\title{
Prevalence, Predictors, and Prevention of Motion Sickness in Zero-G Parabolic Flights
}

\author{
John F. Golding; Aurore C. Paillard; Hervé Normand; Stéphane Besnard; Pierre Denise
}

\begin{abstract}
INTRODUCTION: Zero-G parabolic flight reproduces the weightlessness of space for short periods. However, motion sickness may affect some fliers. The aim was to assess the extent of this problem and to find possible predictors and modifying factors.

METHODS: Airbus zero-G flights consist of 31 parabolas performed in blocks. Each parabola consisted of $20 \mathrm{~s}$ of $0 \mathrm{~g}$ sandwiched by 20 s of hypergravity of $1.5-1.8 \mathrm{~g}$. The survey covered $N=246$ person-flights (193 men, 53 women), ages (M \pm SD) $36.0 \pm$ $11.3 \mathrm{yr}$. An anonymous questionnaire included motion sickness rating ( $1=$ OK to $6=$ vomiting), Motion Sickness Susceptibility Questionnaire (MSSQ), antimotion sickness medication, prior zero-G experience, anxiety level, and other characteristics.

RESULTS: Participants had lower MSSQ percentile scores (27.4 \pm 28.0$)$ than the population norm of 50. Motion sickness was experienced by $33 \%$ and $12 \%$ vomited. Less motion sickness was predicted by older age, greater prior zero-G flight experience, medication with scopolamine, lower MSSQ scores, but not gender or anxiety. Sickness ratings in fliers pretreated with scopolamine (1.81 \pm 1.58$)$ were lower than for nonmedicated fliers $(2.93 \pm 2.16)$, and incidence of vomiting in fliers using scopolamine treatment was reduced by half to a third. Possible confounding factors including age, sex, flight experience, and MSSQ could not account for this.

CONCLUSION: Motion sickness affected one-third of zero-G fliers despite being intrinsically less motion sickness susceptible compared to the general population. Susceptible individuals probably try to avoid such a provocative environment. Risk factors for motion sickness included younger age and higher MSSQ scores. Protective factors included prior zero-G flight experience (habituation) and antimotion sickness medication.
\end{abstract}

KEYWORDS: motion sickness, parabolic, space, vestibular, scopolamine, habituation, age.

Golding JF, Paillard AC, Normand H, Besnard S, Denise P. Prevalence, predictors, and prevention of motion sickness in zero-G parabolic flights. Aerosp Med Hum Perform. 2017; 88(1):1-7.

pace sickness affects over $50 \%$ of astronauts in the first $24-72 \mathrm{~h}$ in space. ${ }^{1}$ An analysis of astronauts who flew in the Space Shuttle indicated that $67 \%$ had some symptoms and $13 \%$ experienced severe sickness. ${ }^{4}$ After this initial exposure most astronauts adapt, although some re-emergence of symptoms may occur on return to the $1 \mathrm{~g}$ environment of Earth, a kind of mal-de-débarquement syndrome. Zero-G parabolic flight reproduces the weightlessness of space for short periods. It has proved an invaluable research tool. The time duration of exposure to an altered force environment in parabolic flight is much shorter than weightlessness in space. Despite this shorter exposure, motion sickness may be a significant problem for some zero-G fliers.

Several mechanisms have been proposed for space motion sickness and by extension for zero-G flight. The fluid shift hypothesis for space motion sickness, i.e., the observed cephalic shift of body fluids to the head, is now largely discounted, ${ }^{11,14}$ although such effects may make astronauts look temporarily younger in the face, notwithstanding possible long-term damaging effects on the ocular nerve. It is now generally accepted that the vestibular system is the key for all motion sickness, including space sickness. The otolith tilt translation hypothesis ${ }^{16}$ would suggest that movement of the head in weightlessness

From the Department of Psychology, Faculty of Science and Technology, University of Westminster, and the Department of Psychology, Open University, Newham Campus, London, UK, and Normandy University, Caen, France.

This manuscript was received for review in June 2016. It was accepted for publication in September 2016.

Address correspondence to: John F Golding, Professor of Applied Psychology, Department of Psychology, Faculty of Science and Technology, University of Westminster 115 New Cavendish Street, London W1W 6UW, United Kingdom; goldinj@wmin.ac.uk. Reprint \& Copyright $\odot$ by the Aerospace Medical Association, Alexandria, VA. DOI: https://doi.org/10.3357/AMHP.4705.2017 
will cause ambiguous interpretations of otolith output, since there can be no tilt and signals will be perceived as translation. Thus the inherent ambiguity of tilt vs. translation perception of otolith signals, normally resolved by the brain through the expectation of low vs. high motion frequencies in the terrestrial environment, ${ }^{8}$ now becomes overwhelming since there is no background gravity vector. Moreover, the absence of the gravity vector will produce what is arguably an additional conflict, that is between otolith and semicircular canal signals under conditions of weightlessness. ${ }^{1}$ It has also been suggested that any asymmetries between otoliths which have been compensated for by neural mechanisms at $1 \mathrm{~g}$ become unmasked under weightlessness, with a consequent mismatch between signals from otoliths in the left and right labyrinths. ${ }^{5}$ Another hypothesis is that, under weightlessness, the otolith hair cell cilia return to their neutral positions, which means that their combined signals will indicate simultaneous multiple possible directions of the gravity vector, producing continuous multiple conflicts. ${ }^{21}$ This is reminiscent of a violation of the third rule of the three general vestibular consistency rules proposed by Stott: ${ }^{20}$ "UtricleSaccule: any sustained linear acceleration is due to gravity, has an intensity of $1 \mathrm{~g}$ and defines 'downwards.'

However, unlike in the weightlessness of space, during zero$\mathrm{G}$ parabolic flight there is an additional provocative stimulus. The hypergravity periods during parabolic flight must also be considered. It has been shown that head movements even when performed only during the hypergravity portions of parabolic flight are sufficient to cause motion sickness. ${ }^{12}$ This is also true of head movements during sustained hypergravity flight. ${ }^{13}$ The latter avoids any confounding due to the nauseogenic effects of Coriolis cross-coupling if sustained hypergravity is induced by centrifuge rotation. The mechanism for this effect is doubtless a canal-otolith conflict due to exposure to a nonterrestrial force background, but where excess rather than reduced otolith signals are the source of the conflict.

This study had two main aims. The first was to assess the current extent of the problem of motion sickness in Airbus zero-G parabolic flights using an anonymous survey to reduce reporting bias. Moreover, most previous studies on this topic have involved relatively small numbers of fliers on parabolic flights and our target was to obtain both a large sample and high response rates to reduce sampling bias. Given a large sample, the second main aim was to find individual motion sickness predictors and to identify possible moderating or protective factors.

\section{METHODS}

\section{Subjects}

Zero-G flier participants were healthy volunteers with healthy vestibular function and not on current medication. They were fully briefed by an information sheet, gave informed consent, and were free to withdraw at any time. Ethical approval was granted by the Ethics Committee (Psychology) of the University of Westminster.

\section{Questionnaire}

The questionnaire was available in both English and French language versions. In order to encourage high participation rates, it was designed to be quick and easy to complete ( $4 \mathrm{~min}$ approximately). It comprised the following items: age and gender demographics; current menstruation (women only); the maximum motion sickness rating experienced during the flight on a validated scale ${ }^{2}(1=\mathrm{OK}, 2=$ initial symptoms, $3=$ mild nausea, $4=$ moderate nausea, $5=$ severe nausea $\& /$ or retching, 6 = vomiting); approximate time of onset of sickness (at beginning, middle, or end of the 31-parabola series); use of antimotion sickness medication (yes/no); write-in box for the name of any drug with example prompts such as 'scopolamine, scopdex, Stugeron, etc.; prior zero-G experience (number of flights); anxiety level during the flight on a 5 -point scale $(0=$ none, $1=$ mild, $2=$ moderate, $3=$ high, $4=$ extreme); when the questionnaire was completed (immediately, $<1 \mathrm{~h}, 1-3 \mathrm{~h},>3 \mathrm{~h}$, following day after the flight); and the short-form Motion Sickness Susceptibility Questionnaire (MSSQ). The MSSQ is a validated questionnaire that reliably predicts motion sickness tolerance from testing in laboratory settings and from vehicular motion $^{7}$ and is also validated for the French language. ${ }^{15}$ The MSSQ is divided into two parts: Part A (MSA) refers to the experience of motion sickness in childhood; Part B (MSB) concerns the last $10 \mathrm{yr}$ of adulthood. Higher scores indicate greater level of susceptibility to motion sickness. It has extensive normative data, enabling it to be converted into population percentiles.

\section{Procedure}

The survey covered $N=246$ person-flights (193 men, 53 women), ages $(\mathrm{M} \pm \mathrm{SD}) 36.0 \pm 11.3 \mathrm{yr}$. Participants were invited to complete an anonymous questionnaire during the return leg of Airbus parabolic zero-G flights. The survey covered a 2-yr time period. Parabolic zero-G flights were performed in a modified Airbus A300 plane run by the Novespace company, flying from Bordeaux-Mérignac, France. Each flight had a pattern in which 31 parabolas were performed in blocks during the middle portion of the flight, which usually lasts around $2.5 \mathrm{~h}$ overall. Each parabola consisted of $20 \mathrm{~s}$ of $0 \mathrm{~g}$ sandwiched by $20 \mathrm{~s}$ of hypergravity periods of $1.5-1.8 \mathrm{~g}$.

\section{Statistical Analysis}

Descriptives of the survey data were produced as means (SD) or percentages as appropriate. Initially the whole correlation matrix was examined to look for relationships between variables. Multivariate analyses were performed to predict motion sickness rating and vomiting. Multiple linear regression using the default enter method was used to produce a predictor model for the dependent variable of sickness rating, i.e., the maximum sickness rating experienced during the flight and to identify significant predictors. Logistic regression was employed to produce a predictor model for the binary variable vomiting vs. no vomiting and to identify significant predictors for vomiting. Analyses of subsets of the data were performed to further clarify the sources of effects. Chi-square, $t$-tests, or Fisher's exact 
tests were employed to further identify significant effects where relevant. All significance values that could be directional from the statistic employed were 2-tailed. The statistical package used was SPSS version 23.

\section{RESULTS}

\section{General Characteristics}

Since the survey was anonymous, it was not possible to be absolutely definitive as to response rates. However, comparison of the numbers of completed questionnaires returned with the numbers of zero-G scientist fliers or flight crews suggested that response rates to completing the questionnaire was approximately $95 \%$. Most respondents reported completing the questionnaire immediately or within a few hours of the flight (Table I).

Motion sickness was experienced by $33 \%$ and $12 \%$ vomited (Table I). Motion sickness began for the majority during the middle or toward the end of the series of parabolas. Flight experience (Table I) of past zero-G flights averaged around eight flights and was rightward skewed by individuals who had flown many times; the median experience of past zero-G flights was two.

Internal consistency of MSSQ was shown by MSA (child) correlating with MSB (adult), as was expected from the psychometric properties of this instrument $(\mathrm{r}=0.66 P<0.0001)$. Participants were less susceptible than the general population, with MSSQ percentile scores of $27.4 \pm 28.0$ vs. a norm of $50 .^{7}$ This finding of reduced inherent susceptibility was similar to that observed in a small earlier survey of zero-G fliers ${ }^{6}$ and is shown in comparison with normative adult samples (see Fig. 1).

Table I. General Characteristics of Zero-G Fliers: Demographics, Motion Sickness and Other Variables as Means (SDs) or Percentages $(N=246)$.

\begin{tabular}{|c|c|}
\hline VARIABLE & MEAN (SD) OR \% \\
\hline Age (years) & $36.0(11.3)$ \\
\hline Sex (Male \%: Female \%) & $78 \% \mathrm{M}: 22 \% \mathrm{~F}$ \\
\hline $\begin{array}{l}\text { Motion sickness rating (scale } 1=\text { OK to } 6= \\
\text { Vomiting) }\end{array}$ & $2.0(1.7)$ \\
\hline Motion sickness: any symptoms (\%) & $33 \%$ \\
\hline Vomited (\%) & $12 \%$ \\
\hline $\begin{array}{l}\text { Onset of motion sickness: beginning; middle; } \\
\text { end of parabolas (\%) }\end{array}$ & $14 \% ; 45 \% ; 41 \%$ \\
\hline $\begin{array}{l}\text { Anxiety during flight (scale } 0=\text { none to } 4= \\
\text { extreme) }\end{array}$ & $0.50(0.67)$ \\
\hline $\begin{array}{l}\text { Flight experience (number of previous zero-G } \\
\text { flights) }\end{array}$ & $7.9(19.1)$ \\
\hline $\begin{array}{l}\text { Antimotion sickness medication taken: Yes \%; } \\
\text { No \% }\end{array}$ & $87 \% ; 13 \%$ \\
\hline $\begin{array}{l}\text { MSA childhood motion sickness susceptibility } \\
\text { (subscale) }\end{array}$ & $4.1(5.0)$ \\
\hline $\begin{array}{l}\text { MSB adult motion sickness susceptibility } \\
\text { (subscale) }\end{array}$ & $2.7(3.9)$ \\
\hline MSSQ motion sickness susceptibility (total score) & $6.8(8.2)$ \\
\hline MSSQ percentile (percentile of population norm) & $27.4(28.0)$ \\
\hline $\begin{array}{l}\text { Questionnaire completed: immediate; }<1 \mathrm{~h} \text {; } \\
1-3 \mathrm{~h} ;>3 \mathrm{~h} \text {; next day }\end{array}$ & $30 \% ; 26 \% ; 23 \% ; 8 \% ; 13 \%$ \\
\hline
\end{tabular}

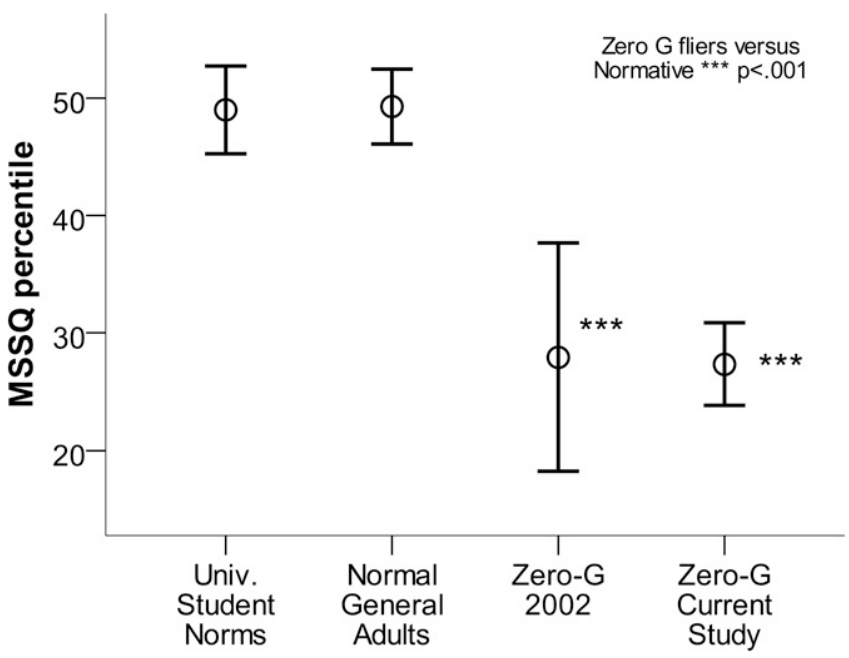

Fig. 1. Motion Sickness Susceptibility Questionnaire (MSSQ) data are shown for normative samples and zero-G fliers for the current study and in the year $2002 .{ }^{6}$ University student normative data $N=257$; general adults normative data $N=$ 395; zero-G 2002 sample $N=23$; current zero-G study $N=249$; error bars are $95 \% \mathrm{Cls}$.

\section{Correlates and Predictors of Motion Sickness}

Significant correlates of motion sickness were as follows. Lower motion sickness ratings were associated with older age, greater prior zero-G flight experience, antimotion sickness medication with scopolamine, lower MSSQ scores, but not with gender or with anxiety (see Table II). In order to see if antimotion sickness medication might act as a distorting factor, these relationships were re-examined for the whole sample after subsetting into those who were medicated vs. unmedicated. The correlations varied somewhat between these subsamples (see Table II), but it should be noted that the unmedicated group size was relatively small when evaluating these associations within each subgroup.

It was noted that there was a significant association between older age and greater previous zero- $G$ flight experience $(\mathrm{r}=$ $0.42, P<0.001)$. In order to attempt some separation of these effects on motion sickness, two types of subanalyses were performed to isolate age effects on motion sickness and then to isolate flight experience effects on motion sickness. The subsample of first time fliers had no prior zero-G flight experience by definition, but the relationship between older age and less motion sickness still remained robust and significant $(\mathrm{r}=$ $-0.36, P<0.001$ ). Then all fliers were subset into younger ( $<$ $30 \mathrm{yr}$ age) and older ( $\geq 30 \mathrm{yr}$ age) groups; this reduced the age with motion sickness correlations to nonsignificance $(\mathrm{r}=$ $-0.12, P=$ n.s.; $\mathrm{r}=-0.18, P=\mathrm{ns}$; respectively). Interestingly, the flight experience with motion sickness correlations remained significant within both of these restricted age range subsamples ( $\mathrm{r}=-0.33, P<0.01 ; \mathrm{r}=-0.28, P<0.01$; respectively). These subanalyses indicated that despite being correlated with each other, both age and flight experience could act independently in their own right as significant variables associated with motion sickness.

Motion sickness rating was predicted using multiple linear regression. The regression model $[F(4)=12.6, P<0.0001$, 
Table II. Correlates of Motion Sickness Rating (Nonparametric Spearman Correlations) for All Zero-G Fliers and Subset by Those with Antimotion Sickness Medication or Not Medicated.

\begin{tabular}{|c|c|c|c|c|c|c|}
\hline \multirow{2}{*}{$\begin{array}{l}\text { PREDICTOR VARIABLE FOR MOTION } \\
\text { SICKNESS RATING }\end{array}$} & \multicolumn{2}{|c|}{$\operatorname{ALL}(N=246)$} & \multicolumn{2}{|c|}{$\begin{array}{l}\text { ANTIMOTION SICKNESS } \\
\text { MEDICATED }(\boldsymbol{N}=\mathbf{2 1 6}) \\
\end{array}$} & \multicolumn{2}{|c|}{$\begin{array}{c}\text { NO MEDICATION } \\
(N=30)\end{array}$} \\
\hline & $\mathbf{r}_{\mathrm{s}}$ & $P$ & $\mathbf{r}_{\mathrm{s}}$ & $P$ & $\mathbf{r}_{\mathrm{s}}$ & $P$ \\
\hline Age & -0.28 & $<0.001$ & -0.20 & $<0.01$ & -0.60 & $<0.001$ \\
\hline Sex & 0.03 & n.s. & 0.04 & n.s. & 0.00 & n.s. \\
\hline Flight Experience & -0.34 & $<0.001$ & -0.27 & $<0.001$ & -0.81 & $<0.001$ \\
\hline Anxiety & 0.09 & n.s. & 0.08 & n.s. & 0.18 & n.s. \\
\hline Antimotion medication & -0.22 & $<0.001$ & --- & --- & --- & --- \\
\hline MSSQ percentile & 0.22 & $<0.001$ & 0.29 & $<0.001$ & 0.03 & n.s. \\
\hline
\end{tabular}

adjusted $\mathrm{R}^{2}=0.17$ ] showed that motion sickness rating could be significantly predicted by the following variables (standardized beta, significance): flight experience $(\mathrm{b}=-0.23, P<$ $0.001)$; age $(\mathrm{b}=-0.14, P<0.05)$; MSSQ $(\mathrm{b}=0.16, P<0.01)$; and antimotion sickness medication $(\mathrm{b}=-0.24, P<0.001)$. All other variables such as gender, anxiety, etc. were not significant predictors. As a check for the robustness of the multiple linear regression model, half of the whole sample was randomly chosen for multiple linear regression. This generated a model which was then used to produce predicted motion sickness values in the other half of the sample. There were no significant observed vs. predicted differences; moreover, the strength of the relationship between observed and predicted motion sickness ratings was significant $(r=0.41, P<0.00012$-tailed) and similar in magnitude to the strength of prediction using the whole sample. This provided support for the robustness of the predictive model. Logistic regression was then used to predict the risk of vomiting as a discrete event. The logistic regression model (omnibus $\chi^{2}=64.7, \mathrm{df}=6, P<0.0001$; Nagelkerke $\mathrm{R}^{2}=0.34$ ) gave $90 \%$ correct classification for vomiting, and showed that vomiting was predicted by the following variables: less flight experience $(P<0.05)$; younger age $(P<0.05)$; and no use of antimotion sickness medication $(P<0.05)$. Although bivariate analysis showed that those who vomited had significantly higher MSSQ scores $(P<0.05)$, MSSQ failed as a vomiting predictor when considered against flight experience, age, and medication in the logistic model. All other variables such as gender, anxiety, etc. were not significant predictors of vomiting.

The following variables showed no useful relationships with motion sickness and were not analyzed further. There was no relationship between motion sickness and when the questionnaires were completed (from immediately postflight through to the following day) (see Table I). There was no significant relationship between the onset time of symptoms of motion sickness and the subsequent overall maximum motion sickness rating (either by correlation or by ANOVA). However, further analysis showed that those who eventually vomited were more likely to have developed motion sickness symptoms earlier at the beginning of the parabola series. Unfortunately, the numbers were too low to satisfy the minimum expected cell requirements of Chi-square, although this result was significant at $P<$ 0.05 using Fisher's exact test. Out of the 53 female fliers,
7 reported that they were menstruating at the time of their zero$\mathrm{G}$ flight. There was no obvious relationship with motion sickness, but numbers were too low for meaningful statistical analysis.

\section{Antimotion Sickness Medication}

All respondents who took antimotion sickness medication who gave further details reported that they took scopolamine and did not report use of other types of medication despite prompts for other types in the free response box. Consequently, although not absolutely definitive due to the anonymous nature of the questionnaire, it is most likely that all or nearly all the antimotion sickness medications taken were scopolamine. Scopolamine was offered as a routine voluntary option to all fliers shortly prior to flight (s.c. injection $0.175 \mathrm{mg}$ scopolamine, but reduced for any individual with low body mass; personal observations indicate that other fliers may have brought and taken their own scopolamine medication as oral pills, taken shortly prior to flight). The mean motion sickness ratings for those who took scopolamine (1.81, SD 1.58) were less than half than those experienced by unmedicated fliers $(2.93$, SD 2.16$)$ and this difference was highly significant $(t=3.5, \mathrm{df}=242, P<0.001$ 2 -tailed). The corresponding effect size of 0.6 for motion sickness rating was 'medium' by common convention, where effect size ' $^{\prime}{ }^{\prime}=($ mean $1-$ mean2) $/($ group SD). The incidence of vomiting in fliers using scopolamine medication was reduced by almost half to a third compared with no medication $\left(\chi^{2}=6.8\right.$, $\mathrm{df}=1, P<0.01)$ (see Fig. 2). This can be stated as a risk ratio for vomiting $=0.38$, medication vs. no medication, i.e., reduced risk with medication. The equivalent risk ratio reduction with scopolamine vs. no medication for occurrence of any motion sickness $=0.49$. Possible confounding factors including age, sex, flight experience, or intrinsic motion sickness susceptibility (MSSQ) were considered. Confounding factors could not account for this scopolamine medication related reduction of motion sickness ratings or lower likelihood of vomiting.

Additional observations on effect sizes of antimotion sickness medication are available for flights surveyed in the year 2002 by the author, ${ }^{6}$ where the medication issued was scopdex (oral combination of scopolamine $0.4 \mathrm{mg}$ and dexamphetamine $5 \mathrm{mg}$ ). Although the numbers were small, the motion sickness ratings were significantly lower in the medicated vs. unmedicated fliers using the same motion rating scale as in the present 


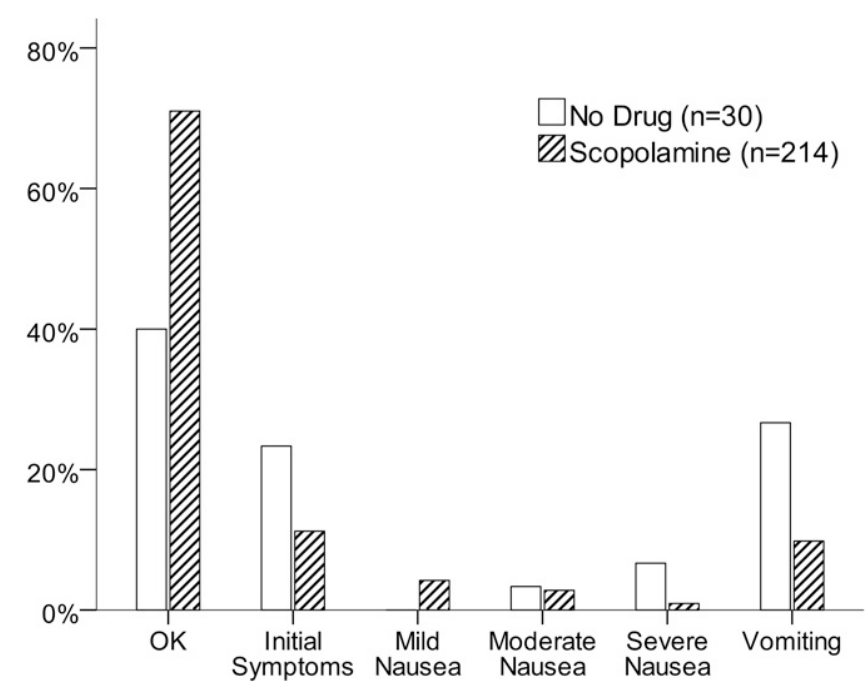

Fig. 2. Motion sickness incidence for fliers treated with the antimotion sickness drug scopolamine vs. untreated 'no drug'. Categories of sickness refer to the maximum sickness experienced. Each flier could only contribute to one category which was the maximum experienced by that individual.

survey (unmedicated $N=7$, ratings $\mathrm{m}=4.43$, $\mathrm{SD} 1.5$; medicated $N=16$, ratings $\mathrm{m}=2.19, \mathrm{SD} 2.0$; significant test difference $t=2.7$, $\mathrm{df}=21, P=0.0142$-tailed). The effect size $(\mathrm{d})$ for the reduction in sickness ratings with medication vs. no medication was $d=1.28$, i.e., somewhat larger for scopdex $(\mathrm{d}=1.28)$ than for scopolamine in the present survey, where $\mathrm{d}=0.6$.

\section{DISCUSSION}

The aims of this study in zero-G fliers were twofold: firstly, to assess the extent of the problem of motion sickness; and secondly, to identify predictors of motion sickness and possible modifying factors. The large size of the sample obtained being well over 200, the high response rate of around 95\%, together with the anonymous nature of the survey to encourage truthful responses, all provided confidence in the accuracy of our estimate of the extent of motion sickness. In zero-G flights 33\% of fliers experienced some degree of motion sickness and $12 \%$ vomited. This is a lower incidence rate than for space sickness, where over $50 \%$ of astronauts have reported experiencing motion sickness in the first $2 \mathrm{~d}$ of weightlessness before they adapt to the new environment. ${ }^{1}$ The higher incidence rates in astronauts may reflect their much greater exposure of days ( $>$ $48 \mathrm{~h}$ ) in an altered force environment. By contrast, the 31 parabolas in a single zero-G flight will only provide an accumulated exposure of alternating $0 \mathrm{~g}$ and $1.5-1.8 \mathrm{~g}$, totaling around $31 \mathrm{~min}$.

Fliers were intrinsically much less motion sickness susceptible compared to the normal population as judged by a validated motion sickness susceptibility questionnaire (MSSQ). ${ }^{7,15}$ Fliers' MSSQ scores showed that their motion sickness susceptibility averaged below the $30^{\text {th }}$ percentile of the general population norms (Fig. 1). This finding of reduced intrinsic motion sickness susceptibility was consistent with observations from a previous but much smaller survey of parabolic flights. ${ }^{6}$ The most likely explanation for this is self-selection. Very susceptible individuals doubtless avoid such a provocative environment if they can. So rather than suggesting that less susceptible individuals are more attracted to undergoing parabolic flights, a better picture is that very highly susceptible individuals know that they are susceptible to motion sickness and consequently will tend to avoid this type of environment if they can.

Two of the most important predictors of increased risk of motion sickness were younger age and less prior experience of zero-G flights. However, these two variables were themselves significantly interrelated, with older fliers being more likely to have had greater prior parabolic flight experience. This is an unsurprising observation since older fliers will have had a greater opportunity for additional zero-G flight experience. But this poses the problem of distinguishing between putative effects of age vs. prior parabolic flight exposure. By selecting subsamples to remove or reduce the effects of either age or flight experience, it was possible to demonstrate that both variables could exert their effects on motion sickness independently of each other. This was also the same picture revealed by multivariate analyses, where both age and flight experience remained significant independent predictors of motion sickness when entered together in the analysis. Correlation does not prove causation, but it seems plausible that both these variables had a causal relationship with motion sickness. Thus, a general reduction in motion sickness susceptibility with age has been observed $^{15}$ and may be related to reduced sensitivity of vestibular functioning, ${ }^{3,17}$ and also perhaps reduced autonomic reactivity with age. ${ }^{18}$ Equally, it is well known that repeated exposures to motion stimuli will cause habituation, which is the most plausible explanation for the reduced risk of motion sickness in those fliers with greater previous zero-G flight experience.

MSSQ scores gave an estimate of intrinsic motion sickness susceptibility for individuals and this was a significant predictor for motion sickness. Higher MSSQ scores significantly predicted greater motion sickness ratings during zero-G parabolic flight and likelihood of vomiting. Although highly statistically significant, the actual magnitude of the strength of this association was less than that observed in previous studies where MSSQ scores have been related with symptom scores and tolerance times to a variety of provocative motion stimuli and transport environments. ${ }^{2,7}$ The most likely explanation for the weaker than expected relationship between MSSQ scores and observed motion sickness is restriction of range in the motion susceptibilities of the sample. As can be seen in Fig. 1, the range of MSSQ scores is very much lower than that expected in the general population. The lack of fliers in the high MSSQ range will have greatly attenuated the possible degree of relationship.

Some variables such as anxiety or gender had little or no significant association with motion sickness. The lack of any great association of anxiety with motion sickness here is perhaps unsurprising. Firstly, reported anxiety levels were very low and this will have had a range restriction effect. Secondly the 
literature on the relationship between anxiety and motion sickness has produced conflicting reports and suggests that such relationships may not be very strong or consistent. ${ }^{15}$ The lack of any significant relationship of motion sickness with gender is more surprising. It is often reported that females tend to be more susceptible. ${ }^{10}$ However, to place this in context it should be noted that the effect of gender is much less than that of age on motion sickness susceptibility. ${ }^{15}$ In space sickness there appears to be a lack of significant differences in susceptibility between genders. ${ }^{1}$ There were relatively few women in the sample compared to men, which reduces the power to find differences. It is also possible that women who are more susceptible have self-selected themselves out; in other words, the selection effect observed for all fliers may have been further enhanced in the women. All of these factors may have contributed to the observed lack of relationship between gender and motion sickness in this survey.

One of the stronger predictors of motion sickness was usage of antimotion sickness medication, in all cases reported to be scopolamine. Motion sickness was significantly lower in those who had taken scopolamine and this could not be explained by any possible confounding factors such as age, sex, prior flight experience, etc. This would suggest (but not prove) a causal effect since scopolamine is regarded as the best proven of all antimotion sickness drugs. ${ }^{19}$ In terms of risk for any motion sickness the risk ratio reduction with scopolamine was 0.49 in zero-G fliers, which is very similar to that proposed in the authoritative Cochrane Review ${ }^{19}$ for the protective actions of scopolamine across a variety of motion environments of 0.48 (95\%CI 0.32-0.73). With regard to vomiting, the risk ratio reduction was 0.38 here, but the Cochrane Review ${ }^{19}$ provides no equivalent estimates for reductions of risk of vomiting by scopolamine vs. no medication, nor for continuous measures of motion sickness. In the present study, the value of the reduction in vomiting risk is particularly useful given the relative lack of data on this metric for protection by scopolamine. Concerning continuous rating measures, the effect size (d) here for scopolamine was a reduction of 0.6 , which is broadly similar to the 0.5 observed in the laboratory using tolerance of cross-coupled motion to levels of moderate nausea. ${ }^{9}$ All of these comparisons provide a practical estimate for the degree of protection that may be provided by scopolamine against motion sickness in the real environment of zero-G flight. This may be summarized as a significant, but far from total protection by scopolamine against motion sickness.

This survey was anonymous, which is both a strength and a possible limitation. Anonymity encourages truthful responses, but had the limitation that we could not identify and select individuals who may have flown for the first time then for all three successive flights over a 1-wk time period (the normal parabolic flight 'campaign'). The identification of the data for such individuals would have been of interest in terms of investigating the dynamics of possible habituation to successive parabolic flights. The questionnaire was kept deliberately short to encourage a high response rate, which was successfully achieved. But this inevitably limited some areas of questioning; for example, we kept the symptom scoring short and simple, which excluded extensive questions on detailed symptoms such as drowsiness or 'sopite.22 Equally detailed questioning of what the flier was doing in term of physically moving around, body postures adopted during zero and hypergravity, head movements, etc. would have been of interest, but would have made the questionnaire longer again. Finally, although this study provided extensive data on motion sickness incidence and on the intrinsic characteristics of fliers, including motion sickness susceptibility, the individual predictors for risk of motion sickness left much unexplained variance, despite being highly significant.

In conclusion, motion sickness affected one-third of zero-G fliers to some extent. Of the zero-G fliers, 1 out of 10 vomited. Fliers were intrinsically less motion sickness susceptible compared to the normal population, perhaps because more motion sickness susceptible individuals try to avoid such a provocative environment if they can. Since this was a survey, the following predictive factors cannot be proven causal, although they are plausible. The main risk factors for motion sickness were younger age and higher MSSQ scores. Protective factors included greater prior zero-G flight experience, probably reflecting habituation and medication prior to the flight with the antimotion sickness drug scopolamine.

\section{ACKNOWLEDGMENTS}

Authors and affiliations: John F. Golding, D.Phil., Department of Psychology, Faculty of Science and Technology, University of Westminster, London, United Kingdom; Aurore C. Paillard, Ph.D. Department of Psychology, Open University, Newham Campus, London, United Kingdom; and Hervé Normand, Ph.D., Stéphane Besnard, Ph.D., and Pierre Denise, Ph.D., Normandie University, UNICAEN, Inserm, Caen, France.

\section{REFERENCES}

1. Benson AJ. Motion sickness. In: Pandolf K, Burr R, editors. Medical aspects of harsh environments, vol. 2. Washington (DC): Walter Reed Army Medical Center; 2002:1060-1094.

2. Bijveld MM, Bronstein AM, Golding JF, Gresty MA. Nauseogenicity of off-vertical-axis rotation versus equivalent visual motion. Aviat Space Environ Med. 2008; 79(7):661-665.

3. Chang CM, Young YH, Cheng PW. Age-related changes in ocular vestibular-evoked myogenic potentials via galvanic vestibular stimulation and bone-conducted vibration modes. Acta Otolaryngol. 2012; 132(12):1295-1300.

4. Davis JR, Vanderploeg JM, Santy PA, Jennings RT, Stewart DF. Space motion sickness during 24 flights of the space shuttle. Aviat Space Environ Med. 1988; 59(12):1185-1189.

5. Diamond SG, Markham CH. Prediction of space motion sickness susceptibility by disconjugate eye torsion in parabolic flight. Aviat Space Environ Med. 1991; 62(3):201-5.

6. Gaudeau C, Golding JF, Lucas Y. Simulating space sickness in the European Space Agency zero-g aircraft. Paper presented at the 8th European Symposium on Life Sciences Research in Space and 23rd Annual International Gravitational Physiology Meeting; 2-7 June 2002; Stockholm, Sweden. Noordwijk (Netherlands): ESA Publication Division; 2002.

7. Golding JF. Predicting individual differences in motion sickness susceptibility by questionnaire. Pers Individ Dif. 2006; 41(2):237-248. 
8. Golding JF, Gresty MA. Biodynamic hypothesis for the frequency tuning of motion sickness. Aerosp Med Hum Perform. 2016; 87(1): 65-68.

9. Golding JF, Stott JRR. Comparison of the effects of a selective muscarinic receptor antagonist and hyoscine (scopolamine) on motion sickness, skin conductance and heart rate. Br J Clin Pharmacol. 1997; 43(6):633-637.

10. Golding JF, Kadzere PN, Gresty MA. Motion sickness susceptibility fluctuates through the menstrual cycle. Aviat Space Environ Med. 2005; 76(10):970-973.

11. Lackner JR, DiZio P. Space motion sickness. Exp Brain Res. 2006; 175(3):377-399.

12. Lackner JR, Graybiel A. Head movements in non-terrestrial force environments elicit motion sickness: implications for the etiology of space motion sickness. Aviat Space Environ Med. 1986; 57(5): 443-448.

13. Muth ER, Raj AK, Rupert AH, Lee R. The experience of nausea during sustained hyper-gravity flight with negligible angular velocity. Aviat Space Environ Med. 2000; 71(5):522-530.

14. Norfleet WT, Coats AC, Powell MR. Inverted immersion as a novel gravitoinertial environment. Aviat Space Environ Med. 1995; 66(9): $825-828$.
15. Paillard AC, Quarck G, Paolino F, Denise P, Paolino M, et al. Motion sickness susceptibility in healthy subjects and vestibular patients: Effects of gender, age and trait-anxiety. J Vestib Res. 2013; 23(4-5):203-209.

16. Parker DE, Reschke MF, Arrott AP, Homick JL, Lichtenberg BK. Otolith tilt-translation reinterpretation following prolonged weightlessness: implications for preflight training. Aviat Space Environ Med. 1985; 56(6):601-606.

17. Rauch SD, Velazquez-Villaseñor L, Dimitri PS, Merchant SN. Decreasing hair cell counts in aging humans. Ann N Y Acad Sci. 2001; 942:220-227.

18. Ray CA, Monahan KD. Aging attenuates the vestibulosympathetic reflex in humans. Circulation. 2002; 105(8):956-961.

19. Spinks A, Wasiak J. Scopolamine (hyoscine) for preventing and treating motion sickness. Cochrane Database Syst Rev. 2011; 6:CD002851.

20. Stott JRR. Mechanisms and treatment of motion illness. In: Davis CJ, Lake-Bakaar GV, Grahame-Smith DG, editors. Nausea and vomiting: mechanisms and treatment. Berlin: Springer-Verlag; 1986:110-129.

21. Thornton WE, Bonato F. Space motion sickness and motion sickness: symptoms and etiology. Aviat Space Environ Med. 2013; 84(7):716-721.

22. Van Ombergen A, Lawson BD, Wuyts FL. Motion sickness and sopite syndrome associated with parabolic flights: a case report. Int J Audiol. 2016; 55(3):189-194 
Author Query sheet-AMHP4705

There are no queries in this article. 